\title{
Intrinsic jet parameters from multifrequency VLA polarization observations of archetypical FR-II quasars and radio galaxies
}

\author{
E. Lüdke ${ }^{1}$, C. V. Andreolla ${ }^{1}$, S. X. Coelho ${ }^{1}$ and O. F. L. Neto ${ }^{1}$ \\ ${ }^{1}$ Universidade Federal de Santa Maria, CCNE - Departamento de Física - LARIE, \\ Campus Universitário, 97150-900 Santa Maria RS, Brazil
}

\begin{abstract}
VLA observations of selected FR-II jets (3C208, 3C352, 3C434, 3C9 and 3C14) between 5 and $15 \mathrm{GHz}$ are used to derive astrophysical properties in the jets of these radio sources. We interpret the results as giving support for unifying schemes of radio galaxies and quasars. We have also determined the Faraday rotation for jets and counter-jet sides. Rotation measures are used to constrain the internal density and mach numbers of the jets, obtaining values similar to those of quasar jets but higher than those of radio galaxies. The radio brightness can be explained by doppler boosting of the synchrotron radiation, giving evidence for orientation effects as an origin for the Laing-Garrington effect.
\end{abstract}

\section{Introduction}

Radio properties are a good indicator of central engine activity in X-ray selected quasars with a single visible jet since there is a tedency of high-power radio-selected DRAGNs to have higher X-ray luminosities (Tananbaum et al. 1993). An evolutionary relationship has also been proposed to unify radiogalaxies and quasars (Harvanek et al. 2001). Evolving radio structures are also expected from Fanaroff-Riley type II objects (FR-II), a few of them with detected superluminal motion within a few parsecs from the core component (Harvanek \& Stocke 2002).

Radio sources with a double structure often show a single jet linking the core to the brightest hostpot in the jet side. A counter-jet side is defined as a region of radio emission opposed to the jet, and a counter-jet is rarely detected. The asymmetry in the jet position and brighness is thought to be due to relativisitic Doppler boosting, as the source is seen at an angle closer than $32^{\circ}$ to the line-of-sight (Barthel 1989).

In this paper we attempt to derive limits for a jet density and kinematical parameters based on arguments of internal depolarization of the jets, using VLA measurements at 5 and $8 \mathrm{GHz}$ of selected radio sources from the revised 3C catalogue (Laing et al. 1983).

\section{The data}

The data comprise VLA observations of selected radio sources at various configurations and epochs, mostly A and B arrays. These sources can be classified as FR-II radio source based on the 6 -cm core luminosity to the integrated $21-\mathrm{cm}$ radio luminosity, according to the Bridle (1986) classification of powerful jets, namely $\mathrm{P}_{\text {core }}^{6 \mathrm{~cm}} \geqslant 10^{23} \mathrm{~W} \mathrm{~Hz}^{-1}$ and $\mathrm{P}_{\text {tot }}^{21 \mathrm{~cm}} \geqslant 10^{24.5} \mathrm{~W} \mathrm{~Hz}^{-1}$.

For the present time, we will limit ourselves to discuss the properties of the selected quasars and radio galaxies shown in figure 1, where radio images of the jet sides are given. A standard circular beam of 350 milliseconds of arc has been used to deconvolve the images to typical noise floors of about $65 \mu \mathrm{Jy}_{\text {beam }}{ }^{-1}$. All these sources show the 
Laing-Garrington effect between 21 and $6 \mathrm{~cm}$ observing wavelength at lower resolutions. The arm length ranges between 12 and $18 \mathrm{kpc}$, with the jet of the largest radio source in our sample, 3C336, having about $28 \mathrm{kpc}$ total length $\left(\mathrm{H}_{0}=50 \mathrm{~km} \mathrm{~s}^{-1} \mathrm{Mpc}^{-1}, q_{0}=1 / 2\right)$.

\section{The results}

Fracional polarizations along the jets are typically $12-22 \%$ for the present sample, whereas the core components of $3 \mathrm{C} 208$ and $3 \mathrm{C} 275.1$ show only marginaly polarized emission $(<4 \%)$. The amount of Faraday rotation between 5 and $8 \mathrm{GHz}$ is very small, as it is the depolarization ratio, suggesting that higher resolutions at 18 and $21 \mathrm{~cm}$ is required to resolve the depolarizing medium in the jets and lobes.

We have run a computer simulation in which tangled magnetic fields with intensities close to equipartition values induce internal depolarization of the synchrotron radiation in cylindrical jets. If the tangling scale is adopted to be about 200 parsecs, we expect the jet to be in equilibrium with the intergalactic medium, if the jet density relative to the IGM particle density is less than $2 \times 10^{-3}$, as expected for light, hypersonic jets. Minimum internal pressures are within the $10^{-11}$ to $10^{-7}$ dyn $\mathrm{cm}^{-2}$. The simulations require also very small particle column densities integrated along the line-of-sight to produce the observed rotation measures.

The jet brightness asymmetries seen in our sample can be explained by standard Doppler boosting analysis, giving the inclination to the line-of-sight angles smaller than $30^{\circ}$, which is consistent with unifying schemes based on relativistic arguments.

Although the jets are not resolved transversally by the VLA, some of them are good targets for polarization-sensitive VLBI observations, to look for superluminal motion at parsec scales.

\section{Acknowledgements}

The Very Large Array national facility is operated by the NRAO on behalf of NSF. Sandro Xavier Coelho and Tina Andreolla thanks CAPES for a research grant. E. Lüdke acknowledges financial support from CNPq and FAPERGS.

\section{References}

Barthel, P. D. 1989, ApJ, 336, 606

Bridle, A. H. 1986, Can. J. Phys., 64, 353

Garrington, S. T., Leahy, J. P., Conway, R. G., \& Laing, R. A. 1988, Nature, 331, 147

Harvanek, M., Ellingson, E., \& Stocke, J. T. 2001, AJ, 122, 2874

Harvanek, M., \& Stocke, J. T. 2002, AJ, 124, 1239

Laing, R. A., Riley, J. M., \& Longair, M. S. 1983, MNRAS, 204, 151

Tananbaum, H., Wardle, J. F. C., Zamorani, G., \& Avni, Y. 1983, ApJ, 268, 60 